\title{
Self-Tuning Control of a Nonlinear Stochastic Systems Described by a Hammerstein Mathematical Model
}

\author{
Houda Salhi \\ Laboratory of Sciences and Techniques of \\ Automatic control \& computer engineering (Lab- \\ STA), University of Sfax, National Engineering \\ School of Sfax Tunisia \\ B.P. 1173, 3038 Sfax-Tunisia
}

\author{
Samira Kamoun \\ Laboratory of Sciences and Techniques of \\ Automatic control \& computer engineering (Lab- \\ STA), University of Sfax, National Engineering \\ School of Sfax Tunisia \\ B.P. 1173,3038 Sfax-Tunisia
}

\begin{abstract}
In this paper, we developed the parametric estimation and the self-tuning control problem of the nonlinear systems which are described by discrete-time nonlinear mathematical models, with unknown, time-varying parameters, and operative in a stochastic environment.

The parametric estimation is realized by using the prediction error method and the recursive least squares techniques. The self-tuning control problem is formulated by minimizing a certain quadratic criterion. An example of numerical simulation is treated in this paper, to test the proposed selftuning control method.
\end{abstract}

\section{General Terms}

Nonlinear systems; Parametric estimation of a stochastic Hammerstein mathematical models; Recursive Instrumental Variable $(R I V)$ algorithm; self-tuning control of a nonlinear system.

\section{Keywords}

Parametric estimation; Discrete-time Hammerstein mathematical model; recursive instrumental variable algorithm; Self-tuning control.

\section{INTRODUCTION}

During these two last decades, a considerable effort was given to the parametric estimation and the control of the complex systems, more particularly, the nonlinear systems operating in a deterministic or stochastic environment.

In this article, we developed the parametric estimation and the self-tuning control problem of the discrete-time nonlinear mathematical models.

The developed work is consists of three parts:

The first part deals the parametric estimation problem of a nonlinear system operated in a stochastic environment. The stress is laid on the class of the nonlinear dynamic systems described by Hammerstein mathematical models with unknown, time-varying parameters. We use the prediction error method and the recursive least squares techniques.

The second part relates to the development of the self-tuning control problem of nonlinear stochastic system that can be described by a Hammerstein stochastic mathematical model. This control problem is formulated while being based on a control strategy minimizing a quadratic criterion. The stress is laid on the class of model where the noise, affecting the measured input value, is correlated with the observations.
Finally, we treat an example of numerical simulation to test the performance of the developed self-tuning control method. The results of numerical simulation obtained are satisfactory.

\section{Parametric estimation of the stochastic}

\section{Hammerstein mathematical model}

The general structure of the Hammerstein mathematical model makes up of a static nonlinear part followed by a dynamic linear part.

The dynamic linear part of the Hammerstein model with timevarying parameters is described by:

$$
A\left(q^{-1}, k\right) y(k)=B\left(q^{-1}, k\right) h(k)
$$

where $y(k)$ and $h(k)$ are respectively the output and the input of the dynamic linear part at the discrete-time $k$ and $A\left(q^{-1}, k\right)$ and $B\left(q^{-1}, k\right)$ are polynomials, which are defined by:

$$
A\left(q^{-1}, k\right)=1+\sum_{i=1}^{n_{A}} a_{i}(k) q^{-i}
$$

and

$$
B\left(q^{-1}, k\right)=\sum_{j=1}^{n_{B}} b_{j}(k) q^{-j}
$$

where $n_{A}$ and $n_{B}$ are the orders of the polynomials $A\left(q^{-1}, k\right)$ and $B\left(q^{-1}, k\right)$, respectively .

The static nonlinear part of the Hammerstein mathematical model is given by the following equation:

$$
h(k)=f_{h}[u(k)]
$$

where $f_{h}[u(k)]$ is a nonlinear function.

The equation (4) can be approximated by a polynomial, that is: $h(k)=\sum_{r=1}^{p} \beta_{r} u^{r}(k)+\Delta h[u(k)]$ 
with $\Delta h[u(k)]$ an approximation error of the nonlinear function, where its variance depends on the selected nonlinearity degree $p$.

Several types of a Hammerstein mathematical models are used in the description of the nonlinear systems, and this, by considering various configurations of the static nonlinear part [1], [2 ] and [ 3 ]. In the case of stochastic systems, two types of Hammerstein mathematical models are known, in order to formulate the parametric estimation problem of the stochastic nonlinear systems. It is about the first type of the Hammerstein mathematical model which is characterized by an input-output mathematical model DARMA, where the noise is correlated with the observations and the second type which is characterize by a linear part described by an input-output ARMAX model with the correlated noise can be described by moving average $M A$ mathematical model (see [3]).

We will interest, in this paper, to the first type of Hammerstein mathematical model.

\subsection{The considered Hammerstein mathematical model}

The considered Hammerstein mathematical model is characterized by an input-output mathematical model $D A R M A$, where the noise, affecting the measured output value, is correlated with the observations.

The structure of this considered mathematical model can be represented by Figure 1.

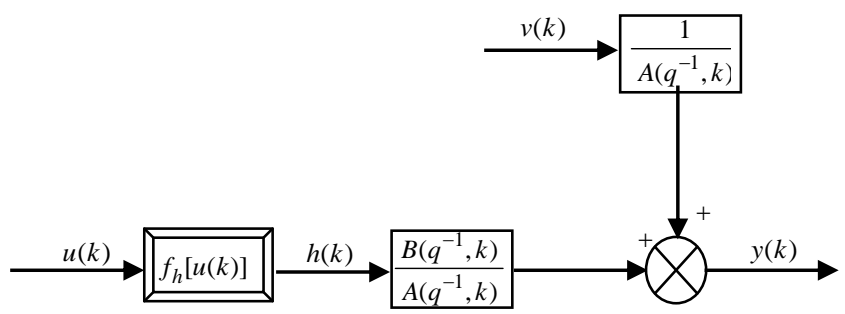

Figure 1. Structure of the considered Hammerstein mathematical model.

According to Figure 2, we can define the linear dynamics part as follows:

$$
A\left(q^{-1}, k\right) y(k)=B\left(q^{-1}, k\right) h(k)+v(k)
$$

where $y(k)$ and $h(k)$ are respectively the output and the input of the dynamic linear block at the discrete-time $k$, $A\left(q^{-1}, k\right)$ and $B\left(q^{-1}, k\right)$ are polynomials defined by the equations (2) and (3), respectively, and $v(k)$ represent the noise (random disturbances), which can act the output system $y(k)$.

the static nonlinear part can be approximated by the polynomial defined by (5).

Finally we can express the output system $y(k)$ in the following form:

$$
y(k)=-\sum_{i=1}^{n_{A}} a_{i}(k) y(k-i)+\sum_{j=1}^{n_{B}} \sum_{r=1}^{p} b_{i}(k) \beta_{r} u^{r}(k-j)+v(k)
$$

The aim had in this part, is the parametric estimation of the mathematical model (7), and this by using an appropriate parametric estimate method.
In order to have parameters estimated without skew, it is necessary to seek a procedure making it possible to eliminate this correlation between the noise and the observations. In this case, the used estimate method is the variable instrumental method with delayed observations, which is based on the modification of the parameters intervening in the observation vector (sequences of the output $y(k)$ and/or of the input $u(k)$ in such way that they are not correlated with the noise sequence $v(k))$.

In order to conclude the synthesis problem of the parametric estimate diagram of the Hammerstein mathematical model, we will suppose, in all follows, that the polynomials $A\left(q^{-1}, k\right)$ and $B\left(q^{-1}, k\right)$ of the linear part of the mathematical model (6) have the same order $n$ (i.e., $\left.n=n_{A}=n_{B}\right)$ and that the parameter $b_{1}(k)$ is well-known at all discrete time $k$. Taking account of these assumptions, we can define the output system $y(k)$ in the following form:

$$
y(k)=\theta^{\mathrm{T}}(k) \psi(k)+v(k)
$$

where $\theta(k)$ represent the parameters vector and $\psi(k)$ indicate the observations vector, which are given by:

$$
\begin{aligned}
\theta^{\mathrm{T}}(k)= & {\left[a_{1}(k), \ldots, a_{n}(k), \beta_{1}, b_{2}(k) \beta_{1} \ldots b_{n}(k) \beta_{1} \ldots \ldots, \beta_{p}\right.} \\
& \left.b_{2}(k) \beta_{p}, \ldots b_{n}(k) \beta_{p}\right]
\end{aligned}
$$

and

$$
\begin{aligned}
\psi^{\mathrm{T}}(k) & =\left[-y(k-1),-\ldots,-y(k-n), u(k-1), \ldots u(k-n), u^{p}(k-1),\right. \\
& \left.\ldots, u^{p}(k-n)\right]
\end{aligned}
$$

Our objective is to estimate the parameters intervening in the vector $\theta(k)$, defined by (9).

The formulation of this parametric estimation problem is made by using the prediction error method which is based on the minimization of a quadratic criterion, based on the difference between the output system and that predicted by the adjustable model, at every discrete-time $k$, by an adaptive mechanism. The a priori predicted output of adjustable model $\hat{y}(k)$ can be defined by the following expression:

$$
\hat{y}(k)=\hat{\theta}^{\mathrm{T}}(k-1) \psi(k)
$$

with $\hat{\theta}(k-1)$ the estimated parameters vector at the discretetime $k-1$, who is given by:

$$
\begin{aligned}
\hat{\theta}^{\mathrm{T}}(k-1)= & {\left[\hat{a}_{1}(k-1), \ldots, \hat{a}_{n}(k-1), \hat{\beta}_{1}(k-1), \hat{b}_{2}(k-1) \hat{\beta}_{1}(k-1) \ldots\right.} \\
& \hat{b}_{n}(k-1) \hat{\beta}_{1}(k-1) \ldots \ldots, \hat{\beta}_{p}(k-1), \\
& \left.\hat{b}_{2}(k-1) \hat{\beta}_{p}(k-1), \ldots \hat{b}_{n}(k-1) \hat{\beta}_{p}(k-1)\right]
\end{aligned}
$$

Then, the a priori prediction error $\varepsilon(k)$ is given by the following expression:

$\varepsilon(k)=y(k)-\hat{\theta}^{\mathrm{T}}(k-1) \psi(k)$

In the instrumental variable method with delayed observations, the parameters intervening in the observations vector $\psi(k)$ are delayed of a certain value. Let us note that the delay value of the observations must be selected in order 
to eliminate the correlation between the observations and the noise $v(k)$

The Recursive Instrumental Variable (RIV) algorithm with forgetting factor is written in the following form:

$$
\begin{aligned}
& \hat{\theta}(k)=\hat{\theta}(k-1)+P(k) z(k) \varepsilon(k) \\
& p(k)=\frac{1}{\lambda(k)}\left[P(k-1)-\frac{P(k-1) z(k) \psi^{\mathrm{T}}(k) P(k-1)}{\lambda(k)+\psi^{\mathrm{T}}(k) P(k-1) z(k)}\right] \\
& \varepsilon(k)=y(k)-\hat{\theta}^{\mathrm{T}}(k-1) \psi(k)
\end{aligned}
$$

where $P(k)$ is a matrix of adaptation gain, which corresponds to the variance-covariance matrix of the noise sequence $v(k)$ $\lambda(k)$ is a forgetting factor $(0 \prec \lambda(k) \prec 1)$ and $z(k)$ is the instrumental vector, which can be given under several alternatives, that more particularly the three following forms:

1. Delay on the output system

$$
\begin{gathered}
z^{\mathrm{T}}(k)=[-y(k-1-t),-\ldots,-y(k-n-t), u(k-1), \ldots u(k-n), \\
\left.u^{p}(k-1), \ldots, u^{p}(k-n)\right]
\end{gathered}
$$

2. Delay on the input and output signals

$$
\begin{aligned}
z^{\mathrm{T}}(k)= & {\left[-y(k-1-t),-\ldots,-y(k-n-t), u\left(k-1-t_{0}\right),\right.} \\
& \left.\ldots u\left(k-n-t_{0}\right), u^{p}\left(k-1-t_{1}\right), \ldots, u^{p}\left(k-n-t_{1}\right)\right]
\end{aligned}
$$

3. Filtering on the output signal

$$
\begin{aligned}
z^{\mathrm{T}}(k)= & {\left[-y_{f}(k-1),-\ldots,-y_{f}(k-n), u(k-1), \ldots u(k-n),\right.} \\
& \left.u^{p}(k-1), \ldots, u^{p}(k-n)\right]
\end{aligned}
$$

where $t, t_{0}$ and $t_{1}$ are selected delay in adequate way and the sequence $\left\{y_{f}(k-r) ; r=1, \ldots, n\right\}$ is obtained by filtering the output system by a certain stable filter having a suitable structure.

The forgetting factor $\lambda(k)$ can be calculated starting from the following recurring equation:

$\lambda(k)=\lambda_{0} \lambda(k-1)+\lambda^{0}\left(1-\lambda_{0}\right)$

with $0<\lambda^{0}<1$ and $0<\lambda_{0}<1$.

The practical implementation of the recursive instrumental variable algorithm $R I V$ requires its initialization. We must thus give the values of the initial conditions of the estimated parameters vector and the adaptation gain matrix.

\section{Self-tuning control of a system describes by a stochastic Hammerstein mathematical model}

The self-tuning control has been a very widespread control strategy in various industrial fields, and this, for several years (see, e.g., [4], [ 5 ]).

We will study, in this part, the self-tuning control problem of the nonlinear stochastic system that can be described by a Hammerstein mathematical model. This control problem is formulated while being based on a control strategy minimizing a quadratic criterion.
Let us consider a nonlinear stochastic system with unknown and time-varying parameters, which can be described by a Hammerstein mathematical model.

The dynamic linear part of this model is described by:

$$
y(k)=\frac{q^{-d} B\left(q^{-1}, k\right)}{A\left(q^{-1}, k\right)} h(k)+v(k)
$$

where $y(k)$ and $h(k)$ are respectively the output and the input of the dynamic linear block at the discrete-time $k$, $A\left(q^{-1}, k\right)$ and $B\left(q^{-1}, k\right)$ are polynomials defined by the equations (2) and (3), respectively, $d$ is a delay and $v(k)$ is a correlated noise, which can be defined by a moving average MA mathematical model, such as:

$$
v(k)=e(k)+c_{1} e(k-1)+\ldots+c_{n_{v}} e\left(k-n_{v}\right)
$$

where $\{e(k)\}$ is an independent random variables sequence of zero average and variance $\sigma^{2}$.

The static nonlinear part of the considered Hammerstein model can be approximated by a polynomial given by:

$$
h(k)=r_{1} u(k)+r_{2} u^{2}(k)+\ldots+r_{p} u^{p}(k)
$$

From the equations (20) and (22), we can express the output system in the following form

$$
\begin{aligned}
y(k)= & \frac{q^{-d} B_{10}\left(q^{-1}, k\right)}{A\left(q^{-1}, k\right)} u(k)+\frac{q^{-d} B_{20}\left(q^{-1}, k\right)}{A\left(q^{-1}, k\right)} u^{2}(k)+\ldots \\
& +\frac{q^{-d} B_{p 0}\left(q^{-1}, k\right)}{A\left(q^{-1}, k\right)} u^{p}(k)+v(k)
\end{aligned}
$$

or in an equivalent way and at the discrete-time $k+d+1$

$$
\begin{aligned}
y(k+d+1) & =\frac{q B_{10}\left(q^{-1}, k\right)}{A\left(q^{-1}, k\right)} u(k)+\frac{q B_{20}\left(q^{-1}, k\right)}{A\left(q^{-1}, k\right)} u^{2}(k)+\ldots \\
& +\frac{q B_{p 0}\left(q^{-1}, k\right)}{A\left(q^{-1}, k\right)} u^{p}(k)+v(k)
\end{aligned}
$$

The problem arising here consists of the development of a regulator which can be applied to the considered stochastic nonlinear system, which is described by the Hammerstein mathematical model (24). The formulation of this regulation problem can be carried out starting from the minimization of the following quadratic criterion $J(k+d+1)$ :

$$
J(k+d+1)=\mathrm{E}\left[y^{2}(k+d+1)\right]
$$

The first stage consists in considering the parameters intervening in the mathematical model (24), recall that the noise is correlated with the observations. Thus, we can use the Recursive instrumental variable algorithm RIV which makes it possible to eliminate this correlation.

Consequently, the calculation of the control law $u(k)$ is based on the knowledge of the estimated parameters of the mathematical model describing this system. The output system $y(k+d+1)$, as defined by (24), can be rewrite as follows, by carrying out an euclidienne division of $1 / A\left(q^{-1}\right)$ until the order $d$ : 


$$
\begin{aligned}
y(k+d+1) & =H_{1}\left(q^{-1}, k\right) u(k)+H_{2}\left(q^{-1}, k\right) u^{2}(k)+\ldots \\
& +H_{p}\left(q^{-1}, k\right) u^{p}(k)+G\left(q^{-1}, k\right) y(k) \\
& +F\left(q^{-1}, k\right) v(k+d+1)
\end{aligned}
$$

where $F\left(q^{-1}, k\right), G\left(q^{-1}, k\right)$ and $H_{i}\left(q^{-1}, k\right), i=1, \ldots, p$, are polynomials defined respectively by:

$$
F\left(q^{-1}, k\right)=1+f_{1}(k) q^{-1}+\ldots+f_{d}(k) q^{-d}
$$

$G\left(q^{-1}, k\right)=g_{0}(k)+g_{1}(k) q^{-1}+\ldots+g_{n-1}(k) q^{-(n-1)}$

and

$$
\begin{aligned}
H_{i}\left(q^{-1}, k\right) & =q \hat{B}_{i 0}\left(q^{-1}, k\right) F\left(q^{-1}, k\right) \\
= & h_{i 1}(k)+h_{i 2}(k) q^{-1}+\ldots+h_{i n+d-1}(k) q^{-n-d+1}
\end{aligned}
$$

with $h_{i 1}(k)=r_{i} b_{1}(k)$.

The two polynomials $F\left(q^{-1}, k\right)$ and $G\left(q^{-1}, k\right)$, such as definite respectively by (27) and (28), are solution of the following polynomial equation:

$1=\hat{A}\left(q^{-1}, k\right) F\left(q^{-1}, k\right)+q^{-d-1} G\left(q^{-1}, k\right)$

We can show easily that the quadratic criterion $J(k+d+1)$, as given by (25), can be put in the following form:

$$
\begin{aligned}
J(k+d+1) & =\left[H_{1}\left(q^{-1}, k\right) u(k)+H_{2}\left(q^{-1}, k\right) u^{2}(k)\right. \\
& \left.+\ldots+H_{p}\left(q^{-1}, k\right) u^{p}(k)+G\left(q^{-1}, k\right) y(k)\right]^{2} \\
& +\left(1+f_{1}^{2}(k)+\cdots+f_{p}^{2}(k)\right) \sigma_{v}^{2} \\
& +2\left(g_{0}^{2}(k)+g_{1}^{2}(k)+\ldots+g_{n}^{2}(k)\left(\left(1+f_{1}^{2}(k)\right.\right.\right. \\
& \left.+\cdots+f_{p}^{2}(k)\right) r_{y v}
\end{aligned}
$$

with $r_{y v}$ is the correlation function between $y(k)$ and $v(k)$, which is defined by :

$r_{y v}=\frac{\operatorname{cov}(y(k), v(k))}{\sigma(y(k)) \sigma(v(k))}$

where $\operatorname{cov}(y(k), v(k))$ is the covariance between the output $y(k)$ and the noise $v(k)$, such that:

$\operatorname{cov}(y(k), v(k))=\frac{\sum_{k=1}^{N} y(k) v(k)}{N}-\bar{y} \bar{v}$

where $(\bar{y}, \bar{v})$ and $(\sigma(y(k)), \sigma(v(k)))$ are, respectively, the averages and the variances of the two signals $y(k)$ and $v(k)$, respectively.

The optimal control law $u(k)$ making it possible to minimize this quadratic criterion is obtained by solving the following equation:

$$
\begin{aligned}
& H_{1}\left(q^{-1}, k\right) u(k)+H_{2}\left(q^{-1}, k\right) u^{2}(k)+\ldots+H_{p}\left(q^{-1}, k\right) u^{p}(k) \\
& +G\left(q^{-1}, k\right) y(k)=0
\end{aligned}
$$

This equation can be put in the following form:

$$
s_{p} u^{p}(k)+\ldots+s_{2} u^{2}(k)+s_{1} u(k)+s_{0}=0
$$

According to the polynomial degree (35), we find $p$ real or complex solutions. In this case, one will hold account only real solutions so that the control law $u(k)$ is physically realizable. If we find more than one real solution, the choice between these various control law $u_{i}(k)$ is done by choosing the control law which checks the following relation:

$\left|u_{i}(k)-u_{00}\right|=\min$

where $u_{00}$ is the control law, which makes it possible to give the desired reference signal .

\section{NUMERICAL SIMULATION}

We treat here an example of numerical simulation of the selftuning control of a nonlinear dynamic stochastic system with unknown time-varying parameters and with a correlated noise. This numerical simulation will be carried out by use the recursive instrumental variable algorithm RIV (15) with forgetting factor. Let us consider a dynamic system being able to be described by the following mathematical model:

$$
A\left(q^{-1}, k\right) y(k)=q^{-1} B\left(q^{-1}, k\right) h(k)+v(k)
$$

The polynomials $A\left(q^{-1}, k\right)$ and $B\left(q^{-1}, k\right)$ are defined by: $A\left(q^{-1}, k\right)=1+a_{1}(k) q^{-1}+a_{2}(k) q^{-2}$

and

$$
B\left(q^{-1}, k\right)=b_{1}(k) q^{-1}+b_{2}(k) q^{-2}
$$

where the time-varying parameters intervening of these two polynomials parameters $A\left(q^{-1}, k\right)$ and $B\left(q^{-1}, k\right)$ are supposed to be unknown and the noise $v(k)$ can be defined by a moving average $M A$ mathematical model, such as:

$v(k)=e(k)+c_{1} e(k-1)$

with $\{e(k)\}$ is an independent random variables sequence of zero average and variance $\sigma^{2}$.

This noise $v(k)$ is supposed to be correlated with the output signal $y(k)$ whose its correlation function is equal to:

$r=0.8670$

and there its covariance $\operatorname{cov}(y(k), v(k))$, is of value:

$\operatorname{cov}(y(k), v(k))=0.2861$

The input $h(k)$ is given by:

$h(k)=r_{1} u(k)+r_{2} u^{2}(k)$ 
We can express the output system $y(k)$ in the following form:

$$
\begin{aligned}
y(k) & =-a_{1}(k) y(k-1)-a_{2}(k) y(k-2)+b_{11}(k) u(k-2) \\
& +b_{12}(k) u(k-3)+b_{21}(k) u^{2}(k-2)+b_{22}(k) u^{2}(k-3) \\
& +v(k)
\end{aligned}
$$

with

$$
\begin{aligned}
& b_{11}(k)=r_{1} * b_{1}(k) \\
& b_{12}(k)=r_{1} * b_{2}(k) \\
& b_{21}(k)=r_{2} * b_{1}(k)
\end{aligned}
$$

and

$$
b_{22}(k)=r_{2} * b_{2}(k)
$$

The mathematical model (44) can be described in the following matric form:

$$
y(k)=\theta^{\mathrm{T}}(k) \psi(k)+e(k)
$$

in which, the parameters vector $\theta(k)$ and the observations vector $\psi(k)$ are defined by:

$\theta^{\mathrm{T}}(k)=\left[a_{1}(k), a_{2}(k), b_{11}(k), b_{12}(k), b_{21}(k), b_{22}(k)\right]$

and

$$
\begin{gathered}
\psi^{\mathrm{T}}(k)=[-y(k-1),-y(k-2), u(k-1), u(k-2), \\
\left.u^{2}(k-1), u^{2}(k-2)\right]
\end{gathered}
$$

We will apply in numerical simulation the control method, presented in section three, with the considered nonlinear system, which is described by the mathematical model (44).

The first stage consists of the parameters estimate $a_{1}(k), a_{2}(k), b_{11}(k), b_{12}(k), b_{21}(k)$ and $b_{22}(k)$ intervening in the mathematical model (44). Thus, we can have at every discrete-time $\hat{a}_{1}(k), \hat{a}_{2}(k), \hat{b}_{11}(k), \hat{b}_{12}(k), \hat{b}_{21}(k)$ and $\hat{b}_{22}(k)$.

The estimate of these parameters is made by the recursive instrumental variable algorithm RIV (15) where the instrumental variable vector is selected in the following form:

$$
\begin{gathered}
z^{\mathrm{T}}(k)=\left[-y_{f}(k-1),-y_{f}(k-2), u(k-2), u(k-3),\right. \\
\left.u^{2}(k-2), u^{2}(k-3)\right]
\end{gathered}
$$

where $\left\{y_{f}(k-r) ; r=1, \ldots, 2\right\}$ is the filtered output obtained by a certain stable filter having the following structure:

$D\left(q^{-1}\right)=1+d_{1} q^{-1}+d_{2} q^{-2}$

where $d_{1}$ and $d_{2}$ are known parameters.

The second step of this self-tuning control problem consists of the solution of a polynomial equation (30); this makes it possible to determine the parameters intervening in the two polynomials $F\left(q^{-1}, k\right)$ and $G\left(q^{-1}, k\right)$, which are given by:

$$
F\left(q^{-1}, k\right)=1+f_{1}(k) q^{-1}
$$

and

$$
G\left(q^{-1}, k\right)=g_{0}(k)+g_{1}(k) q^{-1}
$$

with

$$
\begin{aligned}
& f_{1}(k)=-\hat{a}_{1}(k) \\
& g_{0}(k)=-\hat{a}_{1}(k) f_{1}(k)-\hat{a}_{2}(k)
\end{aligned}
$$

and

$$
g_{1}(k)=-\hat{a}_{2}(k) f_{1}(k)
$$

As for the third step, it relates to the solution of an equation, which is written in the following form:

$z_{2} u^{2}(k)+z_{1} u(k)+z_{0}=0$

with

$$
\begin{aligned}
& z_{2}=\hat{b}_{21}(k) \\
& z_{1}=\hat{b}_{11}(k)
\end{aligned}
$$

and

$$
\begin{aligned}
& z_{0}=h_{11}(k) u(k-1)++h_{12}(k) u(k-2)+h_{21}(k) u^{2}(k-1) \\
& +h_{22}(k) u^{2}(k-2)+g_{0}(k) y(k)+g_{1}(k) y(k-1)
\end{aligned}
$$

with

$$
\begin{aligned}
& h_{11}(k)=\hat{b}_{11}(k) f_{1}(k)+\hat{b}_{12}(k) \\
& h_{12}(k)=\hat{b}_{12}(k) f_{1}(k) \\
& h_{21}(k)=\hat{b}_{21}(k) f_{1}(k)+\hat{b}_{22}(k) ;
\end{aligned}
$$

and

$$
h_{22}(k)=\hat{b}_{22}(k) f_{1}(k)
$$

We now will simulate this method numerically, in order to calculate, with each step of iteration, the value of the control law $u(k)$. For this simulation, we chose the following data:

1. the various parameters values intervening in the equation of the output system $y(k)$, which is given by (44), are chosen, such as:

$$
\begin{aligned}
& \left.a_{1}(k)=-0.8800+0.0300 \sin \mathcal{2} \mathrm{k}\right), \\
& a_{2}(k)=0.4500+0.0200 \sin (\mathrm{k}), \\
& \left.b_{2}(k)=0.3200+0.0200 \sin \mathcal{2}\right), \quad \text { for } \quad k=1 \ldots 200, \\
& b_{1}=1, \beta_{1}=0.43000, \beta_{2}=0.2800 ;
\end{aligned}
$$

2. the estimate of these parameters is made by the recursive instrumental variable algorithm $R I V(15)$ with exponential forgetting factor in the forme (19). The initial conditions of this algorithm are: $\hat{\theta}_{R I V}(0)=0$ and $P_{R I V}(0)=1000 I$, with $I$ is a unit matric ;

3. the reference signal $y_{r}(k)$ to follow being a constant signal of level 20

Let us notice that the estimated parameter $\hat{b}_{2}(k)$ is related to the two estimated parameters $\hat{\beta}_{1}(k)$ and $\hat{\beta}_{2}(k)$. 
we define the estimation error $\delta_{b_{2}}(k)$ of the parameter $b_{2}(k)$ by the following expression:

$\delta_{b_{2}}(k)=b_{2}(k)-\hat{b}_{2 m}(k)$

where $\hat{b}_{2 m}(k)$ is an average estimated, which is given by the following equation:

$\hat{b}_{2 m}(k)=\left(\xi_{21}(k)+\xi_{22}(k)\right) / 2$

where $\xi_{21}(k)$ et $\xi_{22}(k)$ are two estimated parameters, which are given, respectively, by:

$\xi_{21}(k)=\frac{\hat{b}_{12}(k)}{\hat{b}_{11}(k)}$
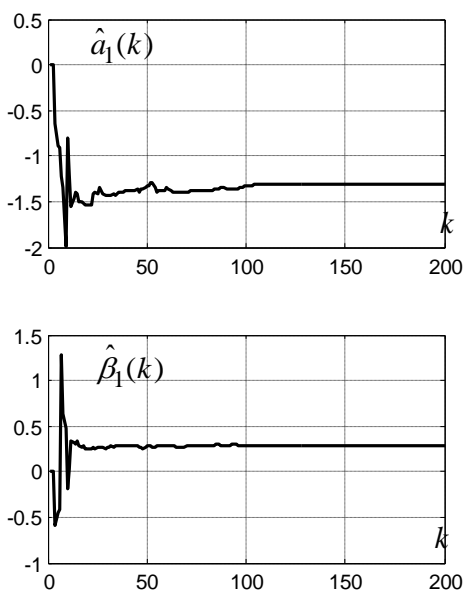

and

$$
\xi_{22}(k)=\frac{\hat{b}_{22}(k)}{\hat{b}_{21}(k)}
$$

To evaluate the parameter estimate quality of $b_{2}(k)$, we propose to calculate the estimation error variance $\delta_{b_{2}}(k)$.

Evolutions curves of the estimated parameters $\hat{a}_{1}(k), \hat{a}_{2}(k)$, $\hat{\beta}_{1}(k)$ and $\hat{\beta}_{2}(k)$ are illustrated in Figure 2.

Figure 3 represents the evolutions curves of the estimated parameters $\hat{b}_{12}(k), \hat{b}_{22}(k)$, of their average value $\hat{b}_{2 m}(k)$ and of the estimation error $\delta_{b_{2}}(k)$ of the parameter $b_{2}(k)$.
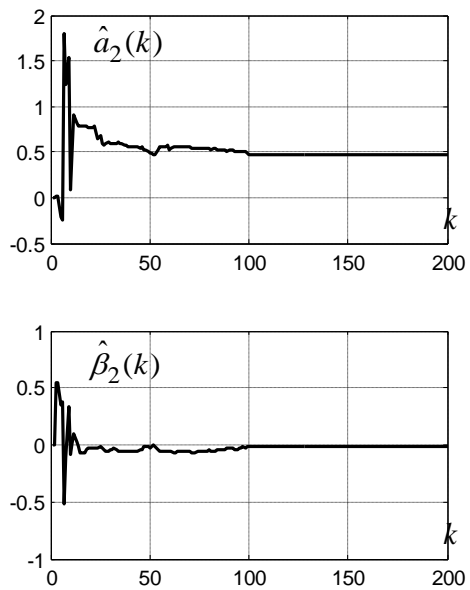

Figure 2. Evolutions curves of the estimated parameters $\hat{a}_{1}(k), \hat{a}_{2}(k), \hat{\beta}_{1}(k)$ and $\hat{\beta}_{2}(k)$


Figure 3. Evolutions curves of the estimated parameters $\hat{b}_{12}(k)$ and $\hat{b}_{22}(k)$, of their average value $\hat{b}_{2 m}(k)$ and of the estimation error $\delta_{b_{2}}(k)$ of the parameter $b_{2}(k)$. 
The statistics average values of the various estimated parameters for $k=101, \ldots, 200$, are given in the following Table 1:

Table 1. Statistics average values of the estimated parameters.

\begin{tabular}{|l|l|l|l|l|l|}
\hline $\bar{m}_{\hat{a}_{1}}$ & $\bar{m}_{\hat{a}_{2}}$ & $\bar{m}_{\hat{\beta}_{11}}$ & $\bar{m}_{\hat{\beta}_{21}}$ & $\bar{m}_{\hat{\beta}_{12}}$ & $\bar{m}_{\hat{\beta}_{22}}$ \\
\hline-0.9814 & 0.4193 & 0.2863 & 0.0469 & 0.2916 & 0.0934 \\
& & & & & \\
\hline
\end{tabular}

The calculation of the statistical average values of the $a$ priori prediction error and his variance are given in the following Table 2.

Table 2. Statistical average values of the prediction error a priori and his variance.

\begin{tabular}{|c|c|}
\hline $\bar{m}_{\varepsilon}$ & $\sigma_{\varepsilon}^{2}$ \\
\hline 0.0107 & 0.0882 \\
\hline
\end{tabular}

From the numerical simulation results, we notice that the estimated parameters converge towards the real parameters with a weak variation. Moreover, the average of the prediction error is weak, like its variance. Let us add that the estimation error variance $\delta_{b_{2}}(k)$ of the parameter $b_{2}(k)$ is equal to 0.0224 . The value of this variance being weak; this
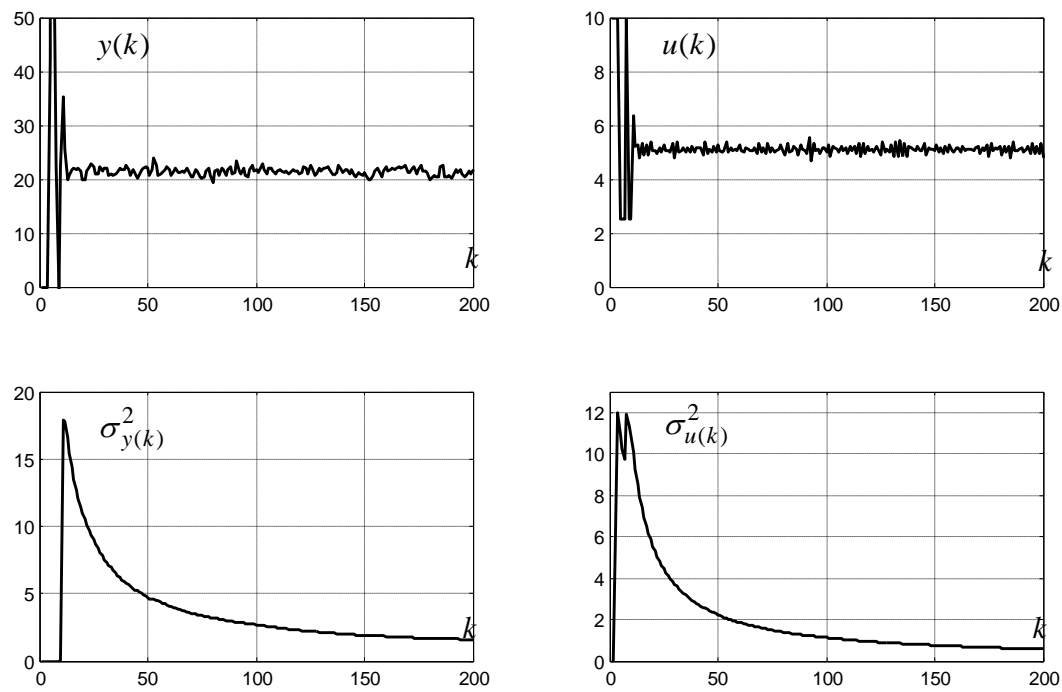

shows the good quality of parameter estimate $b_{2}(k)$.

The obtained results, in this numerical simulation, show well the performances which can ensure the recursive algorithm of parametric estimate $R I V(15)$ during the parameters estimate of a stochastic nonlinear dynamic system describes by a Hammerstein mathematical model with a correlated noise. This good quality of estimate inevitably ensures a good quality of control, and it is to justify, by Figure 4 , which present evolution curves of the output $y(k)$, of control signal $u(k)$, of output variance $\sigma_{y(k)}^{2}$, and of input variance $\sigma_{u(k)}^{2}$.

The output variance $\sigma_{y(k)}^{2}$, and the input variance $\sigma_{u(k)}^{2}$, in permanent mode, can be given in the following table:

Table 3. Output variance $\sigma_{y(k)}^{2}$, and input variance $\sigma_{u(k)}^{2}$

\begin{tabular}{|c|c|}
\hline$\sigma_{y(k)}^{2}$ & $\sigma^{2} u(k)$ \\
\hline 1.1528 & 0.5705 \\
\hline
\end{tabular}

Figure 3 . Evolution curves of the output $y(k)$, of the control signal $u(k)$, of the output variance $\sigma_{y(k)}^{2}$, and of the input variance $\sigma_{u(k)}^{2}$.

\section{CONCLUSION}

In this article, we have developed the parametric estimation and the self-tuning control problem of the discrete-time nonlinear mathematical model, with unknown time-varying parameters, and operative in a stochastic environment. We have used the prediction error method and the recursive least squares techniques.
The stress is laid on the class of the nonlinear stochastic dynamic systems described by a Hammerstein mathematical models type.

\section{ACKNOWLEDGMENTS}

We thank the ministry of higher education and scientific research of Tunisia for funding this work. 


\section{REFERENCES}

[1] Haber, R. and L. Keviczky.1999. Nonlinear System Identification: Input-output Modelling Approach. Kluwer, Boston

[2] Voros, J.2002. Modeling and parameter identification of systems with multisegment piecewise-linear characteristics. IEEE Transactions on Automatic Control, vol. AC-47, pp. 184-188.

[3] Kamoun, S. 2003. Contribution to the identification and the self-tuning control of complex systems. Thesis of doctorate in Electrical Engineering (Automatic), National Engineering School of Sfax, University of Sfax, Tunisie.

[4] Isermann et al.1992. Adaptive Control Systems. Prentice-Hall, New York.
[5] Salhi, H. 2010. Development of the self-tuning control diagrams of the nonlinear systems. Memory of master in automatic and industrial data processing. National Engineering School of Sfax, University of Sfax, Tunisie..

[6] Oliver Nelles . 2001. Nonlinear system identification. Springer, 2001

[7] Laurain.V and al. 2008. Refined Instrumental Variable Methods for Hammerstein Box-Jenkins Models. Proceedings of the $47^{\text {th }}$ IEEE Conference on Decision and Control, Cancun, Mexico.

[8] Wang.X and al. 2011. Identification of Hammerstein Models Based on Online Support Vector Regression. Proceedings of the $30^{\text {th }}$ Chinese Control Conference July 22-24, 2011, Yantai, China. 